\title{
EFFECT OF SOME RESISTANCE INDUCER COMPOUNDS ON QUALITY AND CONTROL OF POSTHARVEST DISEASES OF MANGO FRUITS
}

\author{
H. G. El-Mehrat ${ }^{(1)}$, H. H. Yoness ${ }^{(2)}$ and H. H. Abotaleb(3) \\ (1) Central Lab. of Organic Agriculture, Agricultural Research Center, Giza, Egypt. \\ (2) Department of post Harvest Diseases, Plant Pathology Research Institute, Giza, Egypt. \\ (3) Soil, Water and Environment Institute, Agricultural Res. Center, Giza, Egypt.
}

Received: Nov. 7, 2016

Accepted: Nov. 28,2016

\begin{abstract}
The present study was carried out during the two successive seasons (2014 and 2015) to investigate effectiveness of some resistance inducer compounds on Quality and Control of Postharvest Diseases of Zibda mango fruits under different storage periods. Culciven compound were applied spraying on mango trees at 3 times before harvesting. Mango fruits were harvested at maturity stage then were divided into groups, each group was dipping in one of following materials: Salicylic acid at concentration of 1 and $2 \mathrm{gL}^{-1}$, Potassium Sorbate concentration of 2 and $3 \mathrm{gL}^{-1}$ ) and tape water for 3 minutes and were stored at $10^{\circ} \mathrm{C}$ and $85-$ $90 \% R H$. Samples of fruits were taken weekly during storage periods, to determent weight loss, fruit firmness, fruit decay\% and fruit color as well as total acidity, total soluble solids, total sugars and vitamin $C$ content. The obtained results indicated that the mango trees sprayed with Culciven recorded the best values for all tested fruits parameters as compared to untreated (control). Application of both Salicylic acid and Potassium Sorbate at different concentration supported fruit quality properties. Potassium Sorbate $3 \mathrm{gL}^{-1}$ recorded the higher positive values of weight loss, fruit firmness, fruit decay\%, fruit color, total acidity, total soluble solids, total sugars and vitamin $C$ content as compared to concentration $2 \mathrm{gL}^{-1}$. In contrary, applying Salicylic acid at lower concentration $\left(1 \mathrm{gL}^{-1}\right)$ was the supporter as compared to higher concentration $\left(2 \mathrm{gL}^{-1}\right)$. Moreover, Salicylic acid applied at $1 \mathrm{gL}^{-1}$ concentration gave the best fruit quality as compared to other tested treatments during different storage periods.
\end{abstract}

Key words: Mango, Fruit Quality, Salicylic acid, Potassium Sorbate and Culciven compound

\section{INTRODUCTION}

Mango (Mangifera indicia L.) is one of the most common fruit grown widely in tropical and sub-tropical regions of the world. Mango is known as an appreciable fruit due to its pleasant aroma and flavor, whose nutritional value presents high calories and vitamin contents, among others (Sharma et al., 2006 and Moraeset al., 2010). The fruit is an emerging tropical and sub-tropical export crop produce in about 90 countries (Durraniet al., 2012). The mango world market earns about 700 million dollars per year (FAO, 2010). In Egypt, mango is considered as the third main fruit crop, the fruiting area of mango orchards reached 241101 feddans produced 712537 ton fruits according to the statistics of Ministry of
Agriculture, Egypt (2014). Zibda is greenish the most popular mango (Anacardiceae, Mangifera indicia L.) cultivar in Egypt; this fruit is greenish colored, sweet, low fibercontaining pulp and short shelf life. There are several problems associated with the marketing of mango fruit. Such as the high rate of respiration, moisture loss and susceptibility to microbial attack, especially when ripe, limit the shelf life of mango to less than 10 days, this short shelf life aggravates postharvest losses and does not allow for efficient distribution and marketing (Kolade and Aworh, 1992). The development of postharvest technology related to quality maintenance and extending the postharvest life are an important to consumer acceptability and 
marketing consideration along with export option(Zhong et al., 2006 and Chienet al.,2007).

Effective technologies to ensure a longer shelf-life is desirable with several methods are currently available. Potassium sorbate (KS) is a safe and effective food preservative. It can inhibit effectively the growth of fungi, yeasts, and aerobic bacteria and its toxicity is lower than that of other preservatives, so it has become one of the main preservatives used in the food industry (Karabulut et al., 2001; Karabulut et al., 2005; Fandos and Dominguez, 2007). Salicylic acid (SA) is the plant growth regulator compounds which plays an important role in regulating variety of physiological processes in plants., i, e delaying fruit ripening, softening, and reducing disease resistance as well as reducing disease incidence were discussed by various researchers (Kazemi et al., 2011; Mohamed et al., 2012). Calcium is an important nutrient, plays a crucial role in several physiological functions viz., fruit firmness retention, protecting the plant against salinity stress, callus friability and somatic embryogenesis. Romero et al. (2006) found that the application of calcium as preharvest spray increased fruit quality of mango fruit and Singh et al. (2007) reported that the Calcium nitrate at lower concentration i.e $1.0 \%$ showed beneficial effects in prolonging the storage life of guava fruits. The purpose of the present study was to find out the most suitable postharvest treatments for preservation of mango using available resources by applying some resistance inducers compound.

\section{MATERIALS AND METHODS}

The present work was carried out for two consecutive seasons (2014 and 2015), in a private mango orchard located at ELNobaria district, EL Behira Governorate, where the soil texture is salty, on Zibda mango fruits (Mangifera indicia L.). Eighteen fruitful trees of about 10 years old were selected. The trees were healthy, nearly uniform in their shape, vigor, size and representative for both orchard and cultivar. The selected trees are planted at $3 \times 5$ meters a part. drip irrigation system was applied. The chosen trees were subjected to the normal horticultural practices that are already applied. Spraying with Culciven compound (Cul.), (Culciven, a:i ca $15 \%$ as $\mathrm{CaCl}_{2}$ ) is used at the rate of $1 \mathrm{Liter} / 200 \mathrm{~L}$ water, was done 3 times (15, 30 and 45 days before harvested Mango fruits).

\section{Treatments:-}

The harvested yield from treated trees at maturity stage according to Mohamed, et al. (2008) plus the control fruits collected from untreated trees by Culciven compound (Cul.). The harvested yield divided into 6 groups and treated with Salicylic acid (SA) and Potassium Sorbate (KS)as follows:-

1- Untreated fruits dipping in tape water for 3 minutes (control) (T1).

2- Treated the fruits with Cul. and dipping in tape water for 3 minutes (T2).

3- Treated the fruits with $\mathrm{Cul}$. and dipping in SA $\left(1 \mathrm{~g} / \mathrm{L}^{-1}\right)$ for 3 minutes (T3).

4- Treated the fruits with $\mathrm{Cul}$. and dipping in $\mathrm{SA}\left(2 \mathrm{~g} / \mathrm{L}^{-1}\right)$ for 3 minutes (T4).

5- Treated the fruits with Cul. and dipping in $\mathrm{KS}\left(2 \mathrm{~g} / \mathrm{L}^{-1}\right)$ for 3 minutes (T5).

6 - Treated the fruits with Cul. and dipping in $\mathrm{KS}\left(3 \mathrm{~g} / \mathrm{L}^{-1}\right)$ for 3 minutes (T6).

After subjecting fruits to the above cited treatments, mangoes were dried in air, then placed in carton packages $(5 \mathrm{Kg}$ ) and transferred to cold storage at $10{ }^{\circ} \mathrm{C}$ and $85-$ $90 \% \mathrm{RH}$ at plant pathology Research Institute.

The experimental treatments were arranged in a completely randomized design, with three replicates, each replicate included 3 packages.

Samples were undertaken at 0,7 , $14,21,28$ and 35 days from storage to determine some physical and chemical analysis as follows:- 


\section{Physical properties: \\ 1-Fresh weight \\ Percentage (FW L \%):}

Fresh weight Losses Percentage was calculated as percentage throughout the experimental periods.

\section{2-Fruit Firmness:}

Fruit Firmness was recorded by Lfra texture analyzer instrument using penetrating Cylinderof $5 \mathrm{~mm}$ of diameter to a constant distance $1 \mathrm{~cm}$ inside the fruit using a constant speed at 0,3 $\mathrm{mm} / \mathrm{sec}$, and the results were expressed as the resistance force to the penetrating tester in units of pressure $\mathrm{gm} / \mathrm{mm}^{2}$.

\section{3 - Fruit Decay:}

Decayed fruits due to fungus or any microorganisms' infection was recorded and calculated as a percentage by using the following equation:

Decay\%=weight of decay fruits/initial fruit weight $x 100$.

\section{4 - Fruit colour:}

Skin and flesh colour were measured using a Minolta CR-400 Chroma Meter (Minolta Co .Itd. Osaka, Japan). According to (McGuire, 1992)

\section{Chemical properties:}

Freshly prepared juice of mango fruits samples were used for Total Soluble solids (TSS), total sugars (TS), total acidity as citric acid (TA) and ascorbic acid (V. C) determinations as described by A. O. A.C (2003).

\section{Statistical analysis:}

The obtained data were subjected to analysis of variance according to Snedecor and Cochran (1980) with using SAS package. Comparison of treatment means was done using Tukey test at significance level 0.05 .

\section{Results}

\section{1- Fresh weight Losses \\ Percentage (FW L \%) :-}

Fresh weight losses percentage (FW L) in fruits during different storage period and treated with various levels of the SA or KS as well as Cul. compounds are shown in Table (1). The obtained data clearly showed that significant differences were found between the different applied treatments, the untreated ones recorded the highest FWL \% values as compared to the other treated treatments. For both resistance inducers compound (SA and KS) the FWL \% have lower values as compared to the treatment received (Cul.) as such among the all different storage periods, moreover, the treatments which received SA recorded lower values of FWL \% in comparing with the treatments treated with (KS) in the two applied concentrations, in both seasons and the second season gave lower values for FWL \% as compared to these values having in the first season. On the other hand, SA concentration $\left(1 \mathrm{gL}^{-1}\right)$ was the most effective for FWL \% and delaying ripening mango during storage periods, in compare to other treatments.

\section{2- Fruit Firmness:-}

Data presented in Table (2) illustrated that, mango fruit firmness was decreased with the increasing of storage period and recorded significant differences during the two seasons. The obtained results also indicated that the untreated treatment and/ or treated with (Cul.) as such recorded the lowest mango fruit firmness values as compared to other tested treatments during the two seasons in this work. The treatments which treated with either SA or KS at different concentration scored the highest mango fruit firmness values, moreover, the treatments which received SA $\left(1 \mathrm{gL}^{-1}\right)$ concentration gave higher and significant difference in compare with other all tested treatments. 
El-Mehrat, et al.,

Table 1. Fresh weight Losses Percentage (FW L \%) of Zibda mango fruits during storage periods as affected by application (Cul.), (SA) and (KS) compounds in the two seasons (2014 \& 2015)

\begin{tabular}{|l|c|c|c|c|c|c|c|c|}
\hline \multirow{3}{*}{ Treatments } & \multicolumn{7}{|c|}{ Storage period (weeks) } \\
\cline { 2 - 9 } & 0 & 1 & 2 & 3 & 4 & 5 & mean \\
\cline { 2 - 9 } & \multicolumn{7}{|c|}{ First season } \\
\hline Control & 0.00 & $3.33 \mathrm{a}$ & $6.47 \mathrm{a}$ & $8.20 \mathrm{a}$ & $14.07 \mathrm{a}$ & $20.60 \mathrm{a}$ & $8.78 \mathrm{a}$ \\
\hline Cul. control & 0.00 & $2.93 \mathrm{ab}$ & $5.80 \mathrm{bc}$ & $7.00 \mathrm{bcd}$ & $11.87 \mathrm{~b}$ & $14.40 \mathrm{~b}$ & $7.00 \mathrm{~b}$ \\
\hline SA 1000 ppm & 0.00 & $2.83 \mathrm{~b}$ & $5.53 \mathrm{c}$ & $6.70 \mathrm{~d}$ & $10.80 \mathrm{~cd}$ & $13.00 \mathrm{~d}$ & $6.48 \mathrm{c}$ \\
\hline SA 2000 ppm & 0.00 & $2.83 \mathrm{~b}$ & $5.87 \mathrm{bc}$ & $6.93 \mathrm{~cd}$ & $10.60 \mathrm{~d}$ & $13.03 \mathrm{~cd}$ & $6.54 \mathrm{c}$ \\
\hline KS 2000 ppm & 0.00 & $2.93 \mathrm{ab}$ & $6.20 \mathrm{ab}$ & $7.40 \mathrm{bc}$ & $11.67 \mathrm{bc}$ & $13.03 \mathrm{bcd}$ & $7.00 \mathrm{~b}$ \\
\hline KS 3000 ppm & 0.00 & $3.07 \mathrm{ab}$ & $6.00 \mathrm{abc}$ & $7.53 \mathrm{~b}$ & $12.40 \mathrm{~b}$ & $13.93 \mathrm{bc}$ & $7.15 \mathrm{~b}$ \\
\hline mean & $0.00 \mathrm{f}$ & $2.99 \mathrm{e}$ & $5.98 \mathrm{~d}$ & $7.29 \mathrm{c}$ & $11.90 \mathrm{~b}$ & $14.79 \mathrm{a}$ & 7.16 \\
\hline & \multicolumn{7}{|c|}{ Second season } \\
\hline Control & 0.00 & $3.67 \mathrm{a}$ & $6.17 \mathrm{a}$ & $8.27 \mathrm{a}$ & $13.80 \mathrm{a}$ & $17.80 \mathrm{a}$ & $8.28 \mathrm{a}$ \\
\hline Cul. control & 0.00 & $3.00 \mathrm{bc}$ & $5.40 \mathrm{ab}$ & $7.57 \mathrm{ab}$ & $12.00 \mathrm{ab}$ & $14.07 \mathrm{~b}$ & $7.01 \mathrm{~b}$ \\
\hline SA 1000 ppm & 0.00 & $2.67 \mathrm{c}$ & $3.80 \mathrm{~b}$ & $6.50 \mathrm{~b}$ & $10.43 \mathrm{~b}$ & $11.47 \mathrm{~d}$ & $5.81 \mathrm{c}$ \\
\hline SA 2000 ppm & 0.00 & $2.73 \mathrm{c}$ & $3.93 \mathrm{~b}$ & $7.53 \mathrm{ab}$ & $11.27 \mathrm{~b}$ & $13.20 \mathrm{bc}$ & $6.45 \mathrm{bc}$ \\
\hline KS 2000 ppm & 0.00 & $3.60 \mathrm{a}$ & $5.60 \mathrm{ab}$ & $6.93 \mathrm{ab}$ & $11.66 \mathrm{~b}$ & $12.53 \mathrm{~cd}$ & $6.72 \mathrm{~b}$ \\
\hline KS 3000 ppm & 0.00 & $3.33 \mathrm{ab}$ & $5.20 \mathrm{ab}$ & $6.87 \mathrm{ab}$ & $11.40 \mathrm{~b}$ & $12.73 \mathrm{bcd}$ & $6.59 \mathrm{~b}$ \\
\hline mean & $0.00 \mathrm{f}$ & $3.17 \mathrm{e}$ & $5.02 \mathrm{~d}$ & $7.28 \mathrm{c}$ & $11.76 \mathrm{~b}$ & $13.63 \mathrm{a}$ & 6.81 \\
\hline
\end{tabular}

Means in same column followed by similar letters are not statistically different at 0.05 level according to

$\begin{array}{lll}\text { Tukey test. } & \text { Cul. }=\text { Culciven } & \mathrm{SA}=\text { Salicylic acid } \quad \mathrm{KS}=\text { Potassium Sorbate }\end{array}$

Table 2. Firmness $\left(\mathrm{g} / \mathrm{mm}^{2}\right)$ of Zibda mango fruits during storage periods as affected by application (Cul.), (SA) and (KS) compounds in the two seasons (2014 \& 2015)

\begin{tabular}{|l|c|c|c|c|c|c|c|}
\hline \multirow{3}{*}{ Treatments } & 0 & 1 & 2 & 3 & 4 & 5 & mean \\
\cline { 2 - 8 } & \multicolumn{7}{|c|}{ Storage period (weeks) } \\
\cline { 2 - 8 } & 224.00 & $193.33 \mathrm{c}$ & $167.00 \mathrm{~b}$ & $98.67 \mathrm{c}$ & $56.33 \mathrm{~b}$ & $38.00 \mathrm{c}$ & $129.56 \mathrm{~d}$ \\
\hline Control & 227.33 & $202.67 \mathrm{~b}$ & $198.00 \mathrm{a}$ & $165.00 \mathrm{~b}$ & $109.33 \mathrm{a}$ & $59.00 \mathrm{~b}$ & $160.22 \mathrm{c}$ \\
\hline SA . control $1000 \mathrm{ppm}$ & 227.33 & $213.00 \mathrm{a}$ & $205.33 \mathrm{a}$ & $188.33 \mathrm{a}$ & $119.33 \mathrm{a}$ & $78.33 \mathrm{a}$ & $171.95 \mathrm{a}$ \\
\hline SA 2000 ppm & 227.33 & $215.33 \mathrm{a}$ & $206.00 \mathrm{a}$ & $193.00 \mathrm{a}$ & $117.33 \mathrm{a}$ & $74.00 \mathrm{a}$ & $172.17 \mathrm{a}$ \\
\hline KS 2000 ppm & 227.33 & $210.67 \mathrm{a}$ & $202.00 \mathrm{a}$ & $188.00 \mathrm{a}$ & $113.67 \mathrm{a}$ & $67.67 \mathrm{ab}$ & $168.22 \mathrm{~b}$ \\
\hline KS 3000 ppm & 227.33 & $214.33 \mathrm{a}$ & $202.33 \mathrm{a}$ & $191.00 \mathrm{a}$ & $113.67 \mathrm{a}$ & $70.33 \mathrm{ab}$ & $169.83 \mathrm{ab}$ \\
\hline mean & $226.78 \mathrm{a}$ & $208.22 \mathrm{~b}$ & $196.78 \mathrm{c}$ & $170.67 \mathrm{~d}$ & $104.94 \mathrm{e}$ & $64.56 \mathrm{f}$ & 161.99 \\
\hline & \multicolumn{7}{|c|}{ Second season } \\
\hline Control & $233.33 \mathrm{~b}$ & $194.67 \mathrm{c}$ & $166.67 \mathrm{~d}$ & $94.33 \mathrm{c}$ & $55.33 \mathrm{c}$ & $36.67 \mathrm{~d}$ & $130.17 \mathrm{e}$ \\
\hline Cul. control & $239.33 \mathrm{a}$ & $207.33 \mathrm{~b}$ & $190.67 \mathrm{c}$ & $165.00 \mathrm{~b}$ & $111.33 \mathrm{~b}$ & $65.00 \mathrm{c}$ & $163.11 \mathrm{~d}$ \\
\hline SA 1000 ppm & $239.33 \mathrm{a}$ & $216.00 \mathrm{a}$ & $210.33 \mathrm{a}$ & $189.33 \mathrm{a}$ & $122.00 \mathrm{a}$ & $85.00 \mathrm{a}$ & $177.00 \mathrm{a}$ \\
\hline SA 2000 ppm & $239.33 \mathrm{a}$ & $217.33 \mathrm{a}$ & $207.33 \mathrm{a}$ & $184.33 \mathrm{a}$ & $118.33 \mathrm{ab}$ & $80.00 \mathrm{ab}$ & $174.44 \mathrm{ab}$ \\
\hline KS 2000 ppm & $239.33 \mathrm{a}$ & $215.67 \mathrm{a}$ & $208.00 \mathrm{a}$ & $181.67 \mathrm{a}$ & $116.33 \mathrm{ab}$ & $70.00 \mathrm{c}$ & $171.83 \mathrm{bc}$ \\
\hline KS 3000 ppm & $239.33 \mathrm{a}$ & $210.33 \mathrm{ab}$ & $197.67 \mathrm{~b}$ & $186.00 \mathrm{a}$ & $114.67 \mathrm{ab}$ & $74.00 \mathrm{bc}$ & $170.33 \mathrm{c}$ \\
\hline mean & $238.33 \mathrm{a}$ & $210.22 \mathrm{~b}$ & $196.78 \mathrm{c}$ & $166.78 \mathrm{~d}$ & $106.34 \mathrm{e}$ & $68.45 \mathrm{f}$ & 164.48 \\
\hline
\end{tabular}

Means in same column followed by similar letters are not statistically different at 0.05 level according to Tukey test. $\quad$ Cul. $=$ Culciven $\quad \mathrm{SA}=$ Salicylic acid $\quad \mathrm{KS}=$ Potassium Sorbate 


\section{3- Fruit Decay (\%) of mango fruits:-}

Data in Table (3) clearly showed that decay was observed only in untreated treatment as well as the treatment which treated with Cul. As such on 3 week of storage period and recorded significant difference in the two seasons. Fruits treated by $\mathrm{SA}$ or $\mathrm{KS}$ in the two different concentrations were most effective for delaying ripening of mango and recorded lower values for decay(\%) as compared to other tested treatments but their no significant differences were found between the SA and $\mathrm{KS}$ various concentrations applied in decay(\%) among tested treatments in the two seasons.

\section{4- Color change:-}

Untreated treatment recorded the lowest values for skin colour and flesh colour as compared to other tested treatments during the different storage periods in the two seasons as shown in Tables (4 and 5). The obtained results in Tables (4 and 5) indicated that application of Cul., SA, and Ks at various concentrations did support mango fruits to fully ripe and there is no significance differences were found between various compound applied, but SA in $\left(1 \mathrm{gL}^{-1}\right)$ concentration scored the highest values for skin colour (120.09 and 124.44) and flesh colour (81.15 and 80.81) at first season and second seasons, respectively.

Table 3. Fruit Decay (\%) of Zibda mango fruits as affected by application of some resistance Inducers Compound during storage periods for two seasons (2014 \& 2015)

\begin{tabular}{|c|c|c|c|c|c|c|c|}
\hline \multirow{3}{*}{ Treatments } & \multicolumn{7}{|c|}{ Storage period (weeks) } \\
\hline & 0 & 1 & 2 & 3 & 4 & 5 & mean \\
\hline & \multicolumn{7}{|c|}{ First season } \\
\hline Control & 0.00 & 0.00 & 0.00 & $7.27 \mathrm{a}$ & $11.33 \mathrm{a}$ & $15.07 \mathrm{a}$ & $5.61 \mathrm{a}$ \\
\hline Cul. control & 0.00 & 0.00 & 0.00 & $2.23 \mathrm{~b}$ & $3.93 \mathrm{~b}$ & $6.47 \mathrm{~b}$ & $2.11 \mathrm{~b}$ \\
\hline SA 1000 ppm & 0.00 & 0.00 & 0.00 & $0.00 \mathrm{c}$ & $1.08 \mathrm{c}$ & $2.73 \mathrm{c}$ & $0.64 \mathrm{c}$ \\
\hline SA 2000 ppm & 0.00 & 0.00 & 0.00 & $0.00 \mathrm{c}$ & $1.53 \mathrm{bc}$ & $3.00 \mathrm{c}$ & $0.76 \mathrm{c}$ \\
\hline KS 2000 ppm & 0.00 & 0.00 & 0.00 & $0.00 \mathrm{c}$ & $2.13 \mathrm{bc}$ & $2.73 \mathrm{c}$ & $0.81 \mathrm{c}$ \\
\hline KS 3000 ppm & 0.00 & 0.00 & 0.00 & $0.00 \mathrm{c}$ & $2.00 \mathrm{bc}$ & $2.93 \mathrm{c}$ & $0.82 \mathrm{c}$ \\
\hline \multirow[t]{2}{*}{ mean } & $0.00 \mathrm{~d}$ & $0.00 \mathrm{~d}$ & $0.00 \mathrm{~d}$ & $1.58 \mathrm{c}$ & $3.67 \mathrm{~b}$ & $5.49 \mathrm{a}$ & 1.79 \\
\hline & \multicolumn{7}{|c|}{ Second season } \\
\hline Control & 0.00 & 0.00 & 0.00 & $6.67 \mathrm{a}$ & $10.70 \mathrm{a}$ & $13.83 \mathrm{a}$ & $5.20 \mathrm{a}$ \\
\hline Cul. control & 0.00 & 0.00 & 0.00 & $1.97 \mathrm{~b}$ & $3.40 \mathrm{~b}$ & $7.43 \mathrm{~b}$ & $2.13 \mathrm{~b}$ \\
\hline SA 1000 ppm & 0.00 & 0.00 & 0.00 & $0.00 \mathrm{c}$ & $1.37 \mathrm{bc}$ & $2.63 \mathrm{~cd}$ & $0.77 \mathrm{~cd}$ \\
\hline SA 2000 ppm & 0.00 & 0.00 & 0.00 & $0.00 \mathrm{c}$ & $2.90 \mathrm{bc}$ & $5.60 \mathrm{bc}$ & $1.41 \mathrm{bc}$ \\
\hline KS 2000 ppm & 0.00 & 0.00 & 0.00 & $0.00 \mathrm{c}$ & $1.38 \mathrm{bc}$ & $2.13 \mathrm{~d}$ & $0.59 \mathrm{~cd}$ \\
\hline KS 3000 ppm & 0.00 & 0.00 & 0.00 & $0.00 \mathrm{c}$ & $0.78 \mathrm{c}$ & $2.53 d$ & $0.55 d$ \\
\hline mean & $0.00 \mathrm{~d}$ & $0.00 \mathrm{~d}$ & $0.00 \mathrm{~d}$ & $1.55 \mathrm{c}$ & $3.42 \mathrm{~b}$ & $5.70 \mathrm{a}$ & 1.78 \\
\hline
\end{tabular}

Means in same column followed by similar letters are not statistically different at 0.05 level according to Tukey test.

Cul. = Culciven $\quad$ SA $=$ Salicylic acid

$\mathrm{KS}=$ Potassium Sorbate 
El-Mehrat, et al.,

Table 4. Skin Colour as affected by application of some Resistance Inducers Compound of Zibda mango fruits during storage periods for two seasons (2014 \& 2015)

\begin{tabular}{|l|c|c|c|c|c|c|c|}
\hline \multirow{3}{*}{ Treatments } & \multicolumn{7}{|c|}{ Storage period (weeks) } \\
\cline { 2 - 8 } & 0 & 1 & 2 & 3 & 4 & 5 & mean \\
\cline { 2 - 8 } & \multicolumn{7}{|c|}{ First season } \\
\hline Control & 129.53 & $121.27 \mathrm{~b}$ & $115.80 \mathrm{~b}$ & $112.83 \mathrm{~b}$ & $110.93 \mathrm{~b}$ & $99.67 \mathrm{~b}$ & $115.05 \mathrm{~b}$ \\
\hline Cul. control & 131.23 & $127.53 \mathrm{a}$ & $122.33 \mathrm{a}$ & $118.83 \mathrm{a}$ & $117.03 \mathrm{a}$ & $110.67 \mathrm{a}$ & $121.31 \mathrm{a}$ \\
\hline SA 1000 ppm & 131.23 & $126.90 \mathrm{ab}$ & $120.20 \mathrm{ab}$ & $117.33 \mathrm{a}$ & $114.73 \mathrm{a}$ & $110.00 \mathrm{a}$ & $120.09 \mathrm{a}$ \\
\hline SA 2000 ppm & 131.23 & $125.47 \mathrm{ab}$ & $119.27 \mathrm{ab}$ & $116.63 \mathrm{a}$ & $114.20 \mathrm{a}$ & $109.33 \mathrm{a}$ & $119.34 \mathrm{a}$ \\
\hline KS 2000 ppm & 131.23 & $123.37 \mathrm{ab}$ & $118.63 \mathrm{ab}$ & $116.53 \mathrm{a}$ & $114.27 \mathrm{a}$ & $108.66 \mathrm{a}$ & $118.69 \mathrm{a}$ \\
\hline KS 3000 ppm & 131.23 & $123.43 \mathrm{ab}$ & $122.10 \mathrm{a}$ & $117.70 \mathrm{a}$ & $116.00 \mathrm{a}$ & $108.00 \mathrm{a}$ & $119.73 \mathrm{a}$ \\
\hline mean & $130.95 \mathrm{a}$ & $124.66 \mathrm{~b}$ & $119.72 \mathrm{c}$ & $116.53 \mathrm{~d}$ & $114.53 \mathrm{e}$ & $107.82 \mathrm{f}$ & 119.04 \\
\hline & \multicolumn{7}{|c|}{ Second season } \\
\hline Control & $130.33 \mathrm{a}$ & $126.33 \mathrm{~b}$ & $120.67 \mathrm{~d}$ & $120.00 \mathrm{c}$ & $117.87 \mathrm{~b}$ & $109.13 \mathrm{~b}$ & $120.73 \mathrm{c}$ \\
\hline Cul. control & $133.33 \mathrm{a}$ & $129.67 \mathrm{a}$ & $126.33 \mathrm{a}$ & $124.67 \mathrm{a}$ & $122.47 \mathrm{a}$ & $113.93 \mathrm{a}$ & $125.09 \mathrm{a}$ \\
\hline SA 1000 ppm & $133.33 \mathrm{a}$ & $130.00 \mathrm{a}$ & $125.33 \mathrm{ab}$ & $123.00 \mathrm{ab}$ & $119.37 \mathrm{~b}$ & $115.60 \mathrm{a}$ & $124.44 \mathrm{ab}$ \\
\hline SA 2000 ppm & $133.33 \mathrm{a}$ & $129.66 \mathrm{a}$ & $124.33 \mathrm{~b}$ & $120.67 \mathrm{bc}$ & $118.53 \mathrm{~b}$ & $114.07 \mathrm{a}$ & $123.42 \mathrm{ab}$ \\
\hline KS 2000 ppm & $133.33 \mathrm{a}$ & $129.67 \mathrm{a}$ & $122.67 \mathrm{c}$ & $120.66 \mathrm{bc}$ & $117.20 \mathrm{~b}$ & $113.27 \mathrm{ab}$ & $122.83 \mathrm{~b}$ \\
\hline KS 3000 ppm & $133.33 \mathrm{a}$ & $128.33 \mathrm{ab}$ & $125.00 \mathrm{ab}$ & $123.00 \mathrm{ab}$ & $119.63 \mathrm{ab}$ & $112.70 \mathrm{ab}$ & $123.63 \mathrm{ab}$ \\
\hline mean & $132.78 \mathrm{a}$ & $128.98 \mathrm{~b}$ & $124.12 \mathrm{c}$ & $121.97 \mathrm{c}$ & $119.18 \mathrm{~d}$ & $113.11 \mathrm{e}$ & 123.36 \\
\hline
\end{tabular}

Means in same column followed by similar letters are not statistically different at 0.05 level according to

Tukey test. $\quad$ Cul. $=$ Culciven $\quad \mathrm{SA}=$ Salicylic acid $\quad \mathrm{KS}=$ Potassium Sorbate

Table 5. Flesh Colour as affected by application of some Resistance Inducers Compound of Zibda mango fruits during storage periods for two seasons (2014 \& 2015)

\begin{tabular}{|c|c|c|c|c|c|c|c|}
\hline \multirow{3}{*}{ Treatments } & \multicolumn{7}{|c|}{ Storage period (weeks) } \\
\hline & 0 & 1 & 2 & 3 & 4 & 5 & mean \\
\hline & \multicolumn{7}{|c|}{ First season } \\
\hline Control & $86.73 \mathrm{~b}$ & $82.30 \mathrm{~b}$ & $77.16 \mathrm{~b}$ & $71.07 \mathrm{c}$ & $68.93 c$ & $60.07 \mathrm{~b}$ & $74.38 \mathrm{c}$ \\
\hline Cul. control & $89.10 \mathrm{a}$ & $84.63 \mathrm{ab}$ & $81.17 \mathrm{a}$ & $77.07 \mathrm{~b}$ & $75.77 \mathrm{~b}$ & $69.83 \mathrm{a}$ & $79.59 \mathrm{~b}$ \\
\hline SA 1000 ppm & $89.10 \mathrm{a}$ & $83.77 \mathrm{ab}$ & $82.63 \mathrm{a}$ & $80.18 \mathrm{a}$ & $78.09 a b$ & $73.10 \mathrm{a}$ & $81.15 \mathrm{ab}$ \\
\hline SA $2000 \mathrm{ppm}$ & $89.10 \mathrm{a}$ & $84.03 \mathrm{ab}$ & $81.87 \mathrm{a}$ & $79.63 \mathrm{a}$ & $77.60 \mathrm{ab}$ & $72.20 \mathrm{a}$ & $80.74 \mathrm{ab}$ \\
\hline KS 2000 ppm & $89.10 \mathrm{a}$ & $84.57 \mathrm{ab}$ & $82.53 \mathrm{a}$ & $80.47 \mathrm{a}$ & $77.15 a b$ & $73.37 \mathrm{a}$ & $81.20 \mathrm{ab}$ \\
\hline KS 3000 ppm & $89.10 \mathrm{a}$ & $85.03 \mathrm{a}$ & $82.67 \mathrm{a}$ & $80.98 \mathrm{a}$ & $79.53 \mathrm{a}$ & $71.40 \mathrm{a}$ & $81.45 \mathrm{a}$ \\
\hline \multirow[t]{2}{*}{ mean } & $88.71 \mathrm{a}$ & $84.06 \mathrm{~b}$ & $81.34 \mathrm{c}$ & $78.233 \mathrm{~d}$ & $76.18 \mathrm{e}$ & $69.99 \mathrm{f}$ & 79.75 \\
\hline & \multicolumn{7}{|c|}{ Second season } \\
\hline Control & $88.07 \mathrm{a}$ & $85.13 \mathrm{a}$ & $76.10 \mathrm{c}$ & $71.77 \mathrm{c}$ & $67.50 \mathrm{c}$ & $57.87 \mathrm{c}$ & $74.40 \mathrm{c}$ \\
\hline Cul. control & $89.37 \mathrm{a}$ & $85.23 \mathrm{a}$ & $79.23 \mathrm{bc}$ & $77.50 \mathrm{~b}$ & $74.60 \mathrm{~b}$ & $67.50 \mathrm{ab}$ & $78.90 \mathrm{~b}$ \\
\hline SA 1000 ppm & $89.37 \mathrm{a}$ & $84.03 \mathrm{~b}$ & $81.83 a b$ & $79.93 a b$ & $77.47 \mathrm{a}$ & $72.23 \mathrm{a}$ & $80.81 \mathrm{a}$ \\
\hline SA $2000 \mathrm{ppm}$ & $89.37 \mathrm{a}$ & $84.50 \mathrm{a}$ & $82.30 \mathrm{ab}$ & $78.47 \mathrm{ab}$ & $69.80 \mathrm{c}$ & $66.30 \mathrm{~b}$ & $78.46 \mathrm{~b}$ \\
\hline KS 2000 ppm & $89.37 \mathrm{a}$ & $85.20 \mathrm{a}$ & $83.23 \mathrm{a}$ & $80.43 a b$ & $76.37 \mathrm{ab}$ & $72.30 \mathrm{a}$ & $81.15 \mathrm{a}$ \\
\hline KS 3000 ppm & $89.37 \mathrm{a}$ & $86.37 \mathrm{a}$ & $82.83 \mathrm{a}$ & $81.23 \mathrm{a}$ & $77.90 \mathrm{a}$ & $70.40 a b$ & $81.35 \mathrm{a}$ \\
\hline mean & $89.15 \mathrm{a}$ & $85.08 \mathrm{~b}$ & $80.92 \mathrm{c}$ & $78.22 \mathrm{~d}$ & $73.94 \mathrm{e}$ & $67.76 \mathrm{f}$ & 79.18 \\
\hline
\end{tabular}

Means in same column followed by similar letters are not statistically different at 0.05 level according to

Tukey test. Cul. = Culciven SA = Salicylic acid KS = Potassium Sorbate 


\section{5- Total Acidity (TA):-}

Data in Table (6) indicated that total acidity (TA) was decreased with increasing the storage period in all treatments in the two seasons. The untreated treatment scored significant difference as compared to other tested treatments in both seasons. In contract there are no significant were found among application of various concentration of (Cul.), (SA) and (KS) at total acidity at first season. But at the second season significant difference were found at the treatments which treated with SA as compared to applied Cul. and KS from the first week during the storage periods and the treatment which received SA $\left(1 \mathrm{gL}^{-1}\right)$ recorded the highest value $(0.73 \%)$ for total acidity among all tested treatments.

\section{6- Total Soluble Solids (TSS \%) :-}

In the present study TSS value was increased significantly during the storage periods in all treatments with both SA and $\mathrm{KS}$ at the various concentrations and storage period had also a significant effect on TSS \% in both tested seasons. Data present in Table (7) illustrated that the lowest TSS \% values were recorded in untreated treatment and the treatment which received Cul., as such in compare to other tested treatments the two seasons. The highest TSS \% was found in the treatment which received $\mathrm{KS}$ at $\left(3 \mathrm{gL}^{-1}\right)$ and these values were 13.12 and $13.24 \%$ for the first and second season, respectively. However, TSS \% recorded higher values in the first season as compared to their recorded the second season among the all tested treatments during all different storage periods.

Table 6. Effect of some Resistance Inducers Compound on TA\% of Zibda mango fruits during storage periods for two seasons (2014 \& 2015)

\begin{tabular}{|l|c|c|c|c|c|c|c|c|}
\hline \multirow{3}{*}{ Treatments } & \multicolumn{7}{|c|}{ Storage period (weeks) } \\
\cline { 2 - 8 } & 0 & 1 & 2 & 3 & 4 & 5 & mean \\
\cline { 2 - 9 } & \multicolumn{7}{|c|}{ First season } \\
\hline Control & 1.58 & $0.64 \mathrm{~b}$ & $0.41 \mathrm{~b}$ & $0.15 \mathrm{~b}$ & $0.13 \mathrm{c}$ & $0.11 \mathrm{~b}$ & $0.50 \mathrm{~b}$ \\
\hline Cul. control & 1.60 & $0.84 \mathrm{a}$ & $0.63 \mathrm{a}$ & $0.43 \mathrm{a}$ & $0.34 \mathrm{~b}$ & $0.25 \mathrm{a}$ & $0.68 \mathrm{a}$ \\
\hline SA $1000 \mathrm{ppm}$ & 1.60 & $0.92 \mathrm{a}$ & $0.65 \mathrm{a}$ & $0.45 \mathrm{a}$ & $0.35 \mathrm{ab}$ & $0.27 \mathrm{a}$ & $0.71 \mathrm{a}$ \\
\hline SA 2000 ppm & 1.60 & $0.88 \mathrm{a}$ & $0.64 \mathrm{a}$ & $0.44 \mathrm{a}$ & $0.35 \mathrm{ab}$ & $0.24 \mathrm{a}$ & $0.69 \mathrm{a}$ \\
\hline KS 2000 ppm & 1.60 & $0.84 \mathrm{a}$ & $0.61 \mathrm{a}$ & $0.44 \mathrm{a}$ & $0.38 \mathrm{a}$ & $0.25 \mathrm{a}$ & $0.69 \mathrm{a}$ \\
\hline KS 3000 ppm & 1.60 & $0.85 \mathrm{a}$ & $0.56 \mathrm{a}$ & $0.44 \mathrm{a}$ & $0.37 \mathrm{ab}$ & $0.24 \mathrm{a}$ & $0.68 \mathrm{a}$ \\
\hline mean & $1.60 \mathrm{a}$ & $0.83 \mathrm{~b}$ & $0.58 \mathrm{c}$ & $0.39 \mathrm{~d}$ & $0.32 \mathrm{e}$ & $0.23 \mathrm{f}$ & 0.66 \\
\hline & & & \multicolumn{7}{|c|}{ Second season } & & \\
\hline Control & 1.61 & $0.69 \mathrm{c}$ & $0.42 \mathrm{c}$ & $0.17 \mathrm{c}$ & $0.13 \mathrm{c}$ & $0.12 \mathrm{c}$ & $0.52 \mathrm{e}$ \\
\hline Cul. control & 1.61 & $0.89 \mathrm{~b}$ & $0.68 \mathrm{a}$ & $0.47 \mathrm{ab}$ & $0.30 \mathrm{~b}$ & $0.22 \mathrm{~b}$ & $0.70 \mathrm{~b}$ \\
\hline SA $1000 \mathrm{ppm}$ & 1.61 & $0.96 \mathrm{a}$ & $0.71 \mathrm{a}$ & $0.49 \mathrm{a}$ & $0.35 \mathrm{ab}$ & $0.27 \mathrm{a}$ & $0.73 \mathrm{a}$ \\
\hline SA 2000 ppm & 1.61 & $0.87 \mathrm{~b}$ & $0.61 \mathrm{~b}$ & $0.47 \mathrm{ab}$ & $0.30 \mathrm{~b}$ & $0.23 \mathrm{~b}$ & $0.68 \mathrm{~d}$ \\
\hline KS 2000 ppm & 1.61 & $0.85 \mathrm{~b}$ & $0.63 \mathrm{~b}$ & $0.46 \mathrm{~b}$ & $0.33 \mathrm{ab}$ & $0.26 \mathrm{ab}$ & $0.69 \mathrm{bc}$ \\
\hline KS 3000 ppm & 1.61 & $0.87 \mathrm{~b}$ & $0.60 \mathrm{~b}$ & $0.45 \mathrm{~b}$ & $0.31 \mathrm{~b}$ & $0.26 \mathrm{ab}$ & $0.68 \mathrm{~cd}$ \\
\hline mean & $1.61 \mathrm{a}$ & $0.85 \mathrm{~b}$ & $0.61 \mathrm{c}$ & $0.42 \mathrm{~d}$ & $0.29 \mathrm{e}$ & $0.22 \mathrm{f}$ & 0.67 \\
\hline
\end{tabular}

Means in same column followed by similar letters are not statistically different at 0.05 level according to Tukey test.
Cul. $=$ Culciven
SA = Salicylic acid
$\mathrm{KS}=$ Potassium Sorbate 
El-Mehrat, et al.,

Table 7. Effect of some Resistance Inducers Compound on TSS \% of Zibda mango fruits during storage periods for two seasons (2014 \& 2015)

\begin{tabular}{|c|c|c|c|c|c|c|c|}
\hline \multirow{3}{*}{ Treatments } & \multicolumn{7}{|c|}{ Storage period (weeks) } \\
\hline & 0 & 1 & 2 & 3 & 4 & 5 & mean \\
\hline & \multicolumn{7}{|c|}{ First season } \\
\hline Control & 10.07 & $11.67 \mathrm{bc}$ & $12.47 \mathrm{c}$ & $13.67 \mathrm{ab}$ & $12.47 \mathrm{c}$ & $9.73 \mathrm{~d}$ & $11.68 \mathrm{e}$ \\
\hline Cul. control & 10.30 & $10.73 d$ & $11.90 \mathrm{~d}$ & $13.93 a b$ & $13.73 \mathrm{~b}$ & $12.63 \mathrm{c}$ & $12.20 \mathrm{~d}$ \\
\hline SA 1000 ppm & 10.30 & $10.60 \mathrm{~d}$ & $11.83 \mathrm{~d}$ & $13.00 \mathrm{~b}$ & $14.73 \mathrm{a}$ & $13.73 \mathrm{a}$ & $12.37 \mathrm{~cd}$ \\
\hline SA 2000 ppm & 10.30 & $11.40 \mathrm{c}$ & $12.93 \mathrm{~b}$ & $14.33 \mathrm{a}$ & $14.40 \mathrm{ab}$ & $12.77 \mathrm{c}$ & $12.69 \mathrm{bc}$ \\
\hline KS 2000 ppm & 10.30 & $12.00 \mathrm{ab}$ & $13.27 \mathrm{ab}$ & $14.47 \mathrm{a}$ & $14.77 \mathrm{a}$ & $12.90 \mathrm{bc}$ & $12.95 \mathrm{ab}$ \\
\hline KS 3000 ppm & 10.30 & $12.17 \mathrm{a}$ & $13.57 \mathrm{a}$ & $14.73 \mathrm{a}$ & $14.73 \mathrm{a}$ & $13.20 \mathrm{~b}$ & $13.12 \mathrm{a}$ \\
\hline \multirow[t]{2}{*}{ mean } & $10.26 \mathrm{~d}$ & $11.43 \mathrm{c}$ & $12.66 \mathrm{~b}$ & $14.02 \mathrm{a}$ & $14.14 \mathrm{a}$ & $12.49 \mathrm{~b}$ & 12.50 \\
\hline & \multicolumn{7}{|c|}{ Second season } \\
\hline Control & $9.73 \mathrm{a}$ & $11.73 \mathrm{~b}$ & $12.73 \mathrm{~b}$ & $13.43 \mathrm{~b}$ & $12.80 \mathrm{~b}$ & $9.87 \mathrm{~d}$ & $11.72 \mathrm{e}$ \\
\hline Cul. control & $9.73 \mathrm{a}$ & $10.57 \mathrm{~d}$ & $11.97 \mathrm{c}$ & $14.33 \mathrm{a}$ & $14.33 \mathrm{a}$ & $12.40 \mathrm{c}$ & $12.23 \mathrm{~d}$ \\
\hline SA 1000 ppm & $9.73 \mathrm{a}$ & $10.80 \mathrm{~cd}$ & $12.47 \mathrm{bc}$ & $14.13 \mathrm{ab}$ & $14.80 \mathrm{a}$ & $13.93 \mathrm{a}$ & $12.65 \mathrm{C}$ \\
\hline SA 2000 ppm & $9.73 \mathrm{a}$ & $11.40 \mathrm{bc}$ & $12.67 \mathrm{~b}$ & $14.80 \mathrm{a}$ & $14.50 \mathrm{a}$ & $13.13 \mathrm{a}$ & $12.71 \mathrm{bc}$ \\
\hline KS 2000 ppm & $9.73 \mathrm{a}$ & $11.60 \mathrm{~b}$ & $13.47 \mathrm{a}$ & $14.53 \mathrm{a}$ & $14.87 \mathrm{a}$ & $13.47 \mathrm{~b}$ & $12.95 \mathrm{ab}$ \\
\hline KS 3000 ppm & $9.73 \mathrm{a}$ & $12.37 \mathrm{a}$ & $13.67 \mathrm{a}$ & $14.77 \mathrm{a}$ & $14.97 \mathrm{a}$ & $13.93 \mathrm{a}$ & $13.24 \mathrm{a}$ \\
\hline mean & $9.76 \mathrm{~d}$ & $11.41 \mathrm{c}$ & $12.83 \mathrm{~b}$ & $14.34 \mathrm{a}$ & $14.38 \mathrm{a}$ & $12.79 \mathrm{~b}$ & 12.58 \\
\hline
\end{tabular}

Means in same column followed by similar letters are not statistically different at 0.05 level according to Tukey test.

Cul. = Culciven SA $=$ Salicylic acid

$\mathrm{KS}=$ Potassium Sorbate

\section{7- Total Sugars (TS \%):-}

The data presented in Table (8) rivaled that TS\% significant increasement were found among all tested treatments during the different storage periods the first and second seasons. The treatments which treated with $\mathrm{SA}$ and $\mathrm{KS}$ in the two concentration $\left(1 \& 2 \mathrm{gL}^{-1}\right.$ for $S A$ and $2 \& 3 \mathrm{gL}^{-1}$ for $\mathrm{KS}$ ) having the higher values for TS $\%$ as compared to untreated treatment and/or the treatment which treated by Cul. as such Comparatively higher amount of TS \% was found in all tested treatments at the second season as compared to the first season. The highest total sugar (\%) value was record at the treatment which received $3 \mathrm{gL}^{-1} \mathrm{KS}$ and these values were 11.26 and $11.16 \%$ for second and first seasons respectively. 
Effect of some resistance inducer compounds on quality and

Table 8. Effect of some Resistance Inducers Compound on Total Sugars \% of Zibda mango fruits during storage periods for the two seasons (2014 \& 2015)

\begin{tabular}{|c|c|c|c|c|c|c|c|}
\hline \multirow{3}{*}{ Treatments } & \multicolumn{7}{|c|}{ Storage period (weeks) } \\
\hline & 0 & 1 & 2 & 3 & 4 & 5 & mean \\
\hline & \multicolumn{7}{|c|}{ First season } \\
\hline Control & $8.56 \mathrm{a}$ & $9.92 \mathrm{bc}$ & $10.60 \mathrm{c}$ & $11.62 \mathrm{ab}$ & $10.60 \mathrm{c}$ & $8.27 \mathrm{~d}$ & $9.93 \mathrm{e}$ \\
\hline Cul. control & $8.76 \mathrm{a}$ & $9.12 \mathrm{~d}$ & $10.12 \mathrm{~d}$ & $11.84 \mathrm{ab}$ & $11.67 \mathrm{~b}$ & $10.74 \mathrm{c}$ & $10.38 d$ \\
\hline SA 1000 ppm & $8.76 \mathrm{a}$ & $9.01 \mathrm{~d}$ & $10.06 \mathrm{~d}$ & $11.05 \mathrm{~b}$ & $12.52 \mathrm{a}$ & $11.67 \mathrm{a}$ & $10.51 \mathrm{~cd}$ \\
\hline SA 2000 ppm & $8.76 \mathrm{a}$ & $9.70 \mathrm{c}$ & $10.99 \mathrm{~b}$ & $12.18 \mathrm{a}$ & $12.27 \mathrm{ab}$ & $10.85 \mathrm{c}$ & $10.79 \mathrm{bc}$ \\
\hline KS 2000 ppm & $8.76 \mathrm{a}$ & $10.20 \mathrm{ab}$ & $11.28 \mathrm{ab}$ & $12.57 \mathrm{a}$ & $12.55 \mathrm{a}$ & $10.97 \mathrm{bc}$ & $11.06 \mathrm{ab}$ \\
\hline KS 3000 ppm & $8.76 \mathrm{a}$ & $10.34 \mathrm{a}$ & $11.54 \mathrm{a}$ & $12.53 \mathrm{a}$ & $12.53 \mathrm{a}$ & $11.22 \mathrm{~b}$ & $11.16 \mathrm{a}$ \\
\hline \multirow[t]{2}{*}{ mean } & $8.72 \mathrm{~d}$ & $9.72 \mathrm{c}$ & $10.77 \mathrm{~b}$ & $11.97 \mathrm{a}$ & $12.02 \mathrm{a}$ & $10.62 \mathrm{~b}$ & 10.64 \\
\hline & \multicolumn{7}{|c|}{ Second season } \\
\hline Control & $8.27 \mathrm{a}$ & $9.97 \mathrm{~b}$ & $10.82 \mathrm{~b}$ & $12.20 \mathrm{a}$ & $10.88 \mathrm{~b}$ & $8.39 d$ & $10.09 \mathrm{c}$ \\
\hline Cul. control & $8.30 \mathrm{a}$ & $8.98 \mathrm{~d}$ & $10.29 \mathrm{~b}$ & $11.43 \mathrm{~b}$ & $12.18 \mathrm{a}$ & $10.54 \mathrm{c}$ & $10.29 \mathrm{c}$ \\
\hline SA 1000 ppm & $8.30 \mathrm{a}$ & $9.18 \mathrm{~cd}$ & $10.60 \mathrm{~b}$ & $12.03 \mathrm{ab}$ & $12.58 \mathrm{a}$ & $11.84 \mathrm{a}$ & $10.75 \mathrm{~b}$ \\
\hline SA 2000 ppm & $8.30 \mathrm{a}$ & $9.69 \mathrm{bc}$ & $10.77 \mathrm{~b}$ & $12.60 \mathrm{a}$ & $12.33 \mathrm{a}$ & $11.17 \mathrm{~b}$ & $10.81 b$ \\
\hline KS 2000 ppm & $8.30 \mathrm{a}$ & $9.86 \mathrm{~b}$ & $11.45 \mathrm{a}$ & $12.37 \mathrm{a}$ & $12.64 \mathrm{a}$ & $11.45 b$ & $11.01 \mathrm{ab}$ \\
\hline KS 3000 ppm & $8.30 \mathrm{a}$ & $10.51 \mathrm{a}$ & $11.62 \mathrm{a}$ & $12.57 \mathrm{a}$ & $12.72 \mathrm{a}$ & $11.85 \mathrm{a}$ & $11.26 \mathrm{a}$ \\
\hline mean & $8.30 \mathrm{~d}$ & $9.70 \mathrm{c}$ & $10.92 \mathrm{~d}$ & $12.19 \mathrm{a}$ & $12.22 \mathrm{a}$ & $10.87 \mathrm{~b}$ & 10.70 \\
\hline
\end{tabular}

Means in same column followed by similar letters are not statistically different at 0.05 level according to Tukey test.

Cul. = Culciven

SA = Salicylic acid

$\mathrm{KS}=$ Potassium Sorbate

\section{8-Vitamin C content (V.C $\mathrm{mg} / \mathbf{1 0 0 g}$ pulp):-}

A significant decline in V.C content was found in all tested treatments under Cul., SA and KS components during different storage periods as shown in Table (9). The untreated fruits (control) and/or treated with Cul., as such recorded the lowest values of V.C content in mango fruits and this values were $(36.24 \& 36.83)$ and $(43.29 \& 43.64)$ for control and Cul., treatments in both seasons respectively. On contrary, the highest positive values of V.C content were scored at treatment which received $S A\left(1 \mathrm{gL}^{-1}\right)$ in the second season. Generally, higher values of V.C content fruits content during different storage periods under various applied treatments were recorded in the second season as compared to the first season. 
Table 9. Effect of some Resistance Inducers Compound on Vitamin-C content (V.C $\mathrm{mg} / 100 \mathrm{~g}$ pulp of Zibda mango fruits during storage periods for the two seasons (2014 \& 2015)

\begin{tabular}{|c|c|c|c|c|c|c|c|}
\hline \multirow{3}{*}{ Treatments } & \multicolumn{7}{|c|}{ Storage period (weeks) } \\
\hline & 0 & 1 & 2 & 3 & 4 & 5 & mean \\
\hline & \multicolumn{7}{|c|}{ First season } \\
\hline Control & $54.47 \mathrm{~b}$ & $47.40 \mathrm{~b}$ & $42.47 \mathrm{~d}$ & $38.30 \mathrm{~b}$ & $20.57 \mathrm{c}$ & $14.27 \mathrm{c}$ & $36.24 \mathrm{c}$ \\
\hline Cul. control & $59.47 \mathrm{a}$ & $52.27 \mathrm{a}$ & $49.00 \mathrm{ab}$ & $42.73 \mathrm{a}$ & $33.03 \mathrm{~b}$ & $23.27 \mathrm{~b}$ & $43.29 \mathrm{~b}$ \\
\hline SA 1000 ppm & $59.47 \mathrm{a}$ & $53.47 \mathrm{a}$ & $51.00 \mathrm{ab}$ & $44.00 \mathrm{a}$ & $37.13 \mathrm{ab}$ & $27.13 \mathrm{a}$ & $45.37 \mathrm{a}$ \\
\hline SA 2000 ppm & $59.47 \mathrm{a}$ & $53.87 \mathrm{a}$ & $51.60 \mathrm{a}$ & $45.00 \mathrm{a}$ & $36.53 \mathrm{ab}$ & $26.47 \mathrm{a}$ & $45.49 \mathrm{a}$ \\
\hline KS 2000 ppm & $59.47 \mathrm{a}$ & $53.80 \mathrm{a}$ & $47.73 \mathrm{bc}$ & $43.00 \mathrm{a}$ & $37.80 \mathrm{a}$ & $26.67 \mathrm{a}$ & $44.74 a b$ \\
\hline KS 3000 ppm & $59.47 \mathrm{a}$ & $52.93 \mathrm{a}$ & $47.73 \mathrm{~cd}$ & $43.27 \mathrm{a}$ & $37.53 \mathrm{a}$ & $27.73 \mathrm{a}$ & $44.32 \mathrm{ab}$ \\
\hline \multirow[t]{2}{*}{ mean } & $58.63 \mathrm{a}$ & $52.29 \mathrm{~b}$ & $47.80 \mathrm{c}$ & $42.72 \mathrm{~d}$ & $33.77 \mathrm{e}$ & $24.26 \mathrm{f}$ & 43.24 \\
\hline & \multicolumn{7}{|c|}{ Second season } \\
\hline Control & $56.93 \mathrm{~b}$ & $46.73 \mathrm{~b}$ & $41.77 \mathrm{~d}$ & $38.43 \mathrm{~d}$ & $22.43 \mathrm{~d}$ & $14.67 \mathrm{a}$ & $36.83 \mathrm{c}$ \\
\hline Cul. control & $59.60 \mathrm{a}$ & $52.47 \mathrm{a}$ & $48.27 \mathrm{bc}$ & $41.17 \mathrm{c}$ & $30.67 \mathrm{c}$ & $29.67 \mathrm{~d}$ & $43.64 \mathrm{~b}$ \\
\hline SA 1000 ppm & $59.60 \mathrm{a}$ & $53.80 \mathrm{a}$ & $51.20 \mathrm{ab}$ & $46.50 \mathrm{a}$ & $37.63 \mathrm{ab}$ & $34.07 \mathrm{a}$ & $47.13 \mathrm{a}$ \\
\hline SA 2000 ppm & $59.60 \mathrm{a}$ & $55.13 \mathrm{a}$ & $52.03 \mathrm{a}$ & $45.43 \mathrm{a}$ & $35.23 b$ & $30.60 \mathrm{~cd}$ & $46.34 \mathrm{a}$ \\
\hline KS 2000 ppm & $59.60 \mathrm{a}$ & $53.93 \mathrm{a}$ & $48.20 \mathrm{bc}$ & $43.50 \mathrm{~b}$ & $38.83 \mathrm{a}$ & $31.47 \mathrm{bc}$ & $45.92 \mathrm{a}$ \\
\hline KS 3000 ppm & $59.60 \mathrm{a}$ & $54.13 \mathrm{a}$ & $47.53 \mathrm{c}$ & $44.93 \mathrm{ab}$ & $39.50 \mathrm{a}$ & $32.80 \mathrm{ab}$ & $46.42 \mathrm{a}$ \\
\hline mean & $59.16 \mathrm{a}$ & $52.70 \mathrm{~b}$ & $48.17 \mathrm{c}$ & $43.33 \mathrm{~d}$ & $34.05 \mathrm{e}$ & $28.88 \mathrm{f}$ & 44.38 \\
\hline
\end{tabular}

Means in same column followed by similar letters are not statistically different at 0.05 level according to Tukey test.
Cul. = Culciven
SA = Salicylic acid
$\mathrm{KS}=$ Potassium Sorbate

\section{Discussion}

Pre and postharvest treatments by Cul; SA and KS had a positive effect through storage periods as compared to untreated treatment (control) on biochemical and organoleptic properties of mango fruits. The percentage of Fresh weight losses (FWL\%), Firmness and Decay of mango fruits were recorded expectable values with increasing storage periods during the two seasons at case of application the two inducer compounds salicylic acid (SA) and potassium sorbate(KS). But application of SA was more effective and gave good results as compare to applied KS especially at $1 \mathrm{gL}^{-1} \mathrm{SA}$ concentration. This results is in 
accordance with this obtained by Hemandez-Munoz et al. (2007), Orathai and Lih-shang (2012) and Hossainet al. (2014), they reported that salicylic acid (SA) plays an important role in regulating a variety of physiological processes in plant. The effect of SA on FWL\%, Firmness, delaying fruit ripening softening, increasing disease resistance and reducing disease incidence.

Color changes in mangoes are primarily associated with several biochemical changes, both degradation and synthesis of various classes of molecules including carotenes in fruit. The scores for color changes attained in different treatments with Cul., SA and KS treatments. $\left(1 \mathrm{gL}^{-1}\right) \mathrm{SA}$ concentration as postharvest treatment led to record the significant and higher increases values for mango fruits skin and/or flesh color as compared to other tested treatments, among the two seasons. In this respect our obtained results are in agreement with Yao and Tian (2005), Wang et al. (2006) and Tareenet al.(2012)they found that among different pre and postharvest treatments, the application of SA at lower concentration significantly reduced the change rate of fruits color though different storage periods.

According to Ihekoronye and Ngodd 1985 , total acidity (TA) gives a measure of the amount of acid present in a fruit as citric acid is known to be major acid in mango. Both SA and KS treatments gave a significant effect and higher values in TA as compared to other tested treatments. Moreover SA at $1 \mathrm{gL}^{-1}$ concentration had the good result for TA among the all storage periods tested in the two seasons and reduced the decreases TA as compare to other tested pre and postharvest treatments. These results partially are in harmony with demonstrated by Kazemiet al.(2011), and Tareenet al.(2012) and Hossainet al.(2014)they reported that Apple, Peach and Mango fruits postharvest treated with salicylic acid had significantly higher. TA\% compound with other tested treatments among different storage periods.

Total soluble solids (TSS), Total sugars (TS) and V.C content did effect and exhibit positive change as well as high content of TSS, TS and V.C among different storage periods are an indicator of good quality of mango fruits. In this work, TSS,TS and V.C contents of mango fruits were increased gradually and significantly with the increasing of storage periods during the two seasons under application various concentration of SA and KS as postharvest treatments. These results are in harmony with those obtained by Wang et al.(2006),Barakat et al.(2012), Mohamed et al.(2012), Tareenet al. (2012), Liu et al. (2014) and Reddy \&Sharma (2016)they reported that, application of both salicylic acid and potassium sorbate at low concentration showed beneficial effects in good quality of mango fruits during different storage periods as treated as postharvest treatments. And they added SA and KS were suggested to have the possible influence on fruits metabolic activity leading to great difference in quality properties and physiological characteristics, and these included TSS, TS and V.C.

\section{Conclusion:-}

Postharvest treatment of lower concentration $\left(1 \mathrm{gL}^{-1}\right)$ of salicylic acid (SA) and higher concentration $\left(3 \mathrm{gL}^{-1}\right)$ potassium Sorbate were found to be the most effective treatments among all other treatments on biochemical and organoleptic properties of mango in relation to delay ripening without any external decay.

\section{REFERENCES}

Association of Official Agricultural Chemists, (2003). Official Methods of Analysis (A.O.A.C), 12th Ed., Published by A. O. A. C., Washington D. C., U. S. A.

Barakat, M. R., M. A. A. Mohamed, M. A. Essa and Z.A. Zaki, (2012). Effect of Using Some Biological Post Harvest 
Treatments on Storability of Washington Navel Orange Fruits Compared with Imazalil Post Harvest Chemical Treatments. Journal of Horticultural Science \& Ornamental Plants, 4(1): 5057.

Chien, P. J., F. Sheu and F. H. Yang (2007). Effects of edible chitosan coating on quality and shelf life of sliced mango fruit. J. Food Eng., 78, 225- 229.

Durrani, Y., A. Zeb, Y. Xingqian, M. Ayub, W. Tahir, A. Muhammad and S. Wahab (2012). Influence of storage temperatures on physicochemical sensory and nutritional properties of chemically preserved mango pulp. Afric. J. Biotech, 11(13): 3147- 3152.

Fandos, G. E. and J. L. Dominguez (2007). Effect of potassium sorbate washing on the growth of Listeriamonocytogenes on fresh poultry. Food Control, 18(7): 842846.

Food and Agricultural Organization (FAO), CCP: BA/RF 09/CRS2, December 7, 2010. Joint meeting of the fourth session of the sub-group on bananas and the fifth session of the sub-group on tropical fruits. Tropical fruits compendium.

Hemandez-Munoz, E., V. D. Almenar, D. V. Velez and R. Gavara (2007). Effect of chitosan coating combined with postharvest calcium treatment on strawberry (Fragariaananassa) quality during refrigerated storage. Food Chem., 110(2): 428- 435.

Hossain, F., A. K. Parvez, M. K. Munshi, I. Khalil and R. Huque (2014). Post-harvest treatments of radiation and chemical on organoleptic and biochemical properties of mango (Mangiferaindica L.)In relation to delay ripening. Am-Eur. J. Agric. Environ. Sci., 14(6): 555- 564.

Ihekoronye, A. I. and P. O. Ngoddy (1985). Integrated Food Science and Technology for the tropics. Macmillan publishers Ltd, London.

Karabulut, O. A., G. Romanazzi, J. L. Smilanick and A. Lichter (2005). Postharvest ethanoland potassium sorbate treatments of table grapes to control gray mold. Postharvest Biol. Technol. 37(2): 129-134.

Karabulut, O. A., S. Lurie and S. Droby (2001). Evaluation of the use of sodium bicarbonate, potassium sorbate and yeast antagonists for decreasing postharvest decay of sweet cherries. Postharvest Biol. Technol. 23(3): 233236.

Kazemi, M., M. Aran and S. Zamani (2011). Effect of Salicylic Acid Treatments on Quality Characteristics of Apple Fruits during Storage. American Journal of Plant Physiology, 6, 113-119.

Kolade, J. and O. C. Aworh (1992). Postharvest treatment of wild mango (Irvingiagabonesis) for improved shelf life Food Chemistry, 44(1): 45-48.

Liu Kun, X. W. and M. Young (2014). Effect of bentonite/potassium sorbate coatings on the quality of mangos in storage at ambient temperature. Journal of Food Engineering 137, 16- 22.

McGuire, R. G. (1992). Reporting of objective colour measurements. Hort. Sci., 27(12):1254-1255.

Ministry of Agriculture and land reclamation, Economic affairs sector, Bulletin of the agricultural statistics Part (2) summer\& Neil crops, published in October 2014.

Mohamed, M. A., A. A. Gobara, A. M. A. Ragab and R. T Mouftah (2008). Response of Taimour and Zibda mango trees to application of organic and biofertilization along with seaweed extract. 1st Inter. Conf. on Environ. Studies\& Res. 7 - 9 April, Minufiya Univ. Sadat Branch. pp $28-38$.

Mohamed, M. A. A., H. G. El-Mehrat and A. S. E. Salem (2012). Effect of Postharvest Treatments with Salicylic Acid and Chitocare on Navel Orange Fruits Quality and Storability Compared with the Commercial Postharvest Fungicide Treatment. J. Plant Production, Mansoura Univ., 3(12): 3069 - 3082.

Moraes, I. C. F., R. M. Sampaio, N. M. Queiroz, S. M. Salles, C. C. Pashoaleti 
and V. H. Parez (2010). Mango pulp (Mangiferaindica L.) preserved by hurdle technology: physicochemical, microbiological and rheological characterization. J. Food Proc. And preserve, 35(5): 610- 614.

Orathai, W. and K. Lih-shang (2012). The quality maintenance and extending storage life of mango fruit after postharvest treatments. World Academy of Science, Engineering and Technology, 69, 936- 941.

Reddy, V. R. and R. R. Sharma (2016). Effect of pre-harvest application of salicylic acid on postharvest fruit quality of the Amrapali mango (Mangiferaindica). Indian Journal of Agricultural Sciences, 86(6): 727- 731.

Romero, N. R., C. Saucedo, P. Garcia, J. Alcazar, V. M. Hernandez and R. Sanudo (2006). Foliar application of calcium: Physiology and fruit quality of Haden mango. Terra, 24(4): 521-527.

Sharma, G. P., M. Dak and R. C. Verma (2006). Flow characteristics of juice of "Totapuri" mangoes. J. Food Eng. 76(4): 557- 561.

Singh, R., O. P. Chaturvedi, G. S. Gaur and G. Singh (2007). Effect of preharvest spray of zinc, calcium and boron on the storage behavior of guava fruits cv. Allahabad safeda. Acta Hort., 735, 633638

Snedecor, G. W. and W. G. Cochran (1980). Statistical methods. 7th edition lowa State Univ. Press Ames., Iowa, U.S.A. p. 593.

Tareen, M. J., N. A. Abbasi and S. Hafeez (2012). Effect of salicylic acid treatments on storage life of Peach fruits cv. 'flordaking'. Pak. J. Bot., 44(1):119-124.

Wang, L., S. Chena, W. Kong, S. Li and D. D. Archbold (2006). Salicylic acid pretreatment alleviates chilling injury and affects the antioxidant system and heat shock proteins of peaches during cold storage. Postharvest Biol. and Technol., 41, 244-251.

Yao, H. and S. Tian (2005). Effects of preand post-harvest application of salicylic acid or methyl jasmonate on inducing disease resistance of sweet cherry fruit in storage. Postharvest Biology and Technology, 35, 253-262..

Zhong, Q. P., W. S. Xia and Y. Jiang (2006). Effects of 1-methylcyclopropene treatments on ripening and quality of harvested sapodilla fruit. Food Technoland Biotechnol, 44(4): 535- 539 
تأثير بعض المركبات المستحثة علي جودة و مقاومة أمراض ما بعد الحصاد لثمار المانجو

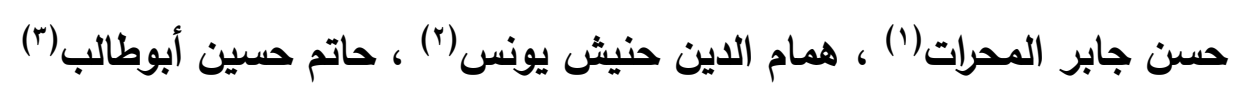

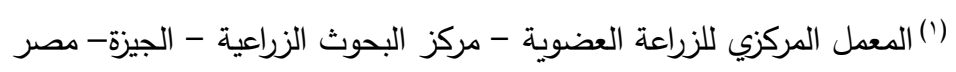

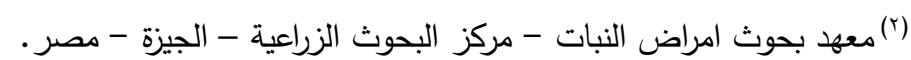

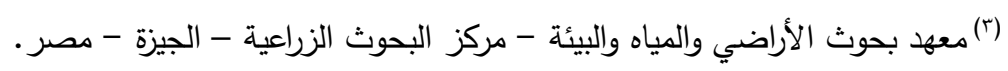
الملخص العربي

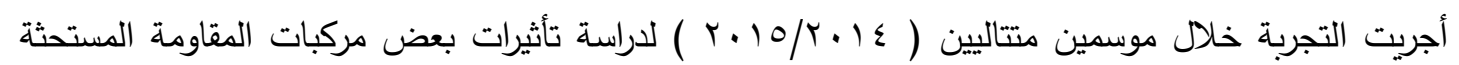


الكالسفين رشا على اشجار المانجو ثلاث مرات قبل الحصاد و تم حصاد ثمار المانجو في طور اكتمال النمو وتم

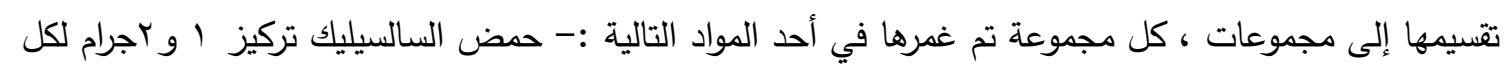

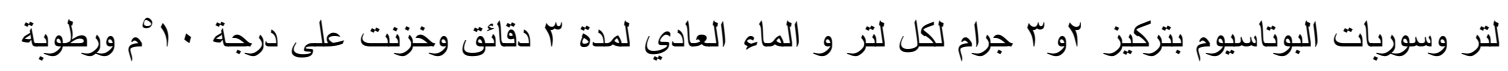

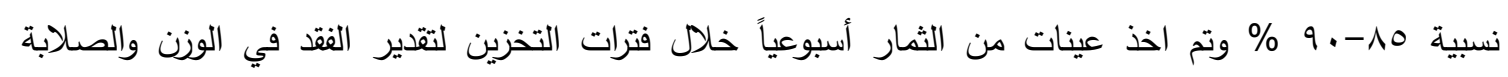
والإصابة المرضية واللون وكذلك الحموضة الكلية والمواد الصلبة الذائبة والسكريات الكلية وفيتامين ج.

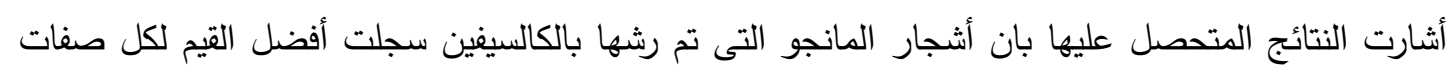

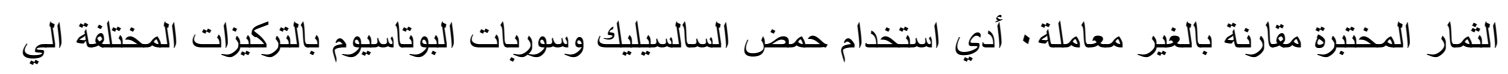

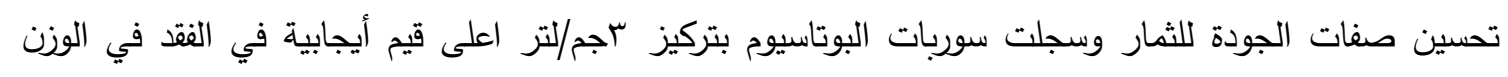

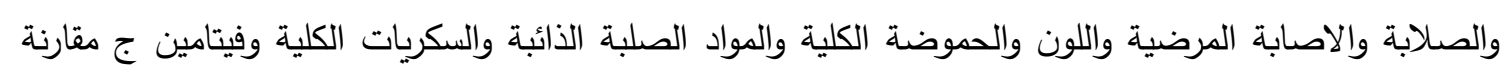
بتركيز rجم / لتر وعلى العكس من ذلك استخدام حامض السالسيليك بالتركيز المنخفض (اجم / لتر) كان متفوقاً

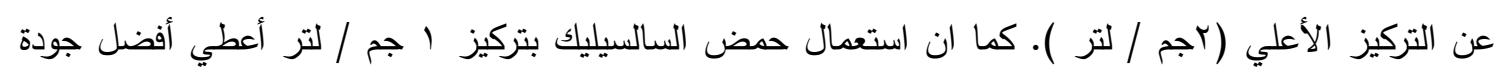

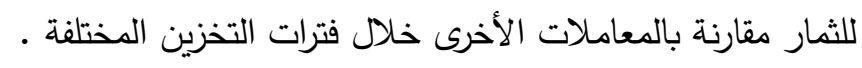

